tion is relatively greater than it was before the cornea was incised, we must endeavor by counter-pressure and immobility of the eye to hasten the re-adhesion and firmness of the corneal wound; in one word, to restore as rapidly as possible the equilibrium between external and internal pressure. Great differences of opinion exist regarding the after-treatment. Chisholm stands at the head of a faction who oppose bandages and restraint of all kinds. He simply fastens the lids of the operated eye by gold-beater's skin or a piece of adhesive plaster, leaves the other eye open and gives the patient, protected only by dark glasses, the freedom of the ward. Others, again, permit the bandaged patient to walk from the operating room to his bed and there remain quietly for two days. Some operators do not remove the first dressing until forty-eight hours elapse; others operate on the patient in bed so as to avoid any possible strain attributed to locomotion and undressing. I heartily agree with the innovators of the idea that patients should be free from the restraint which was formerly placed upon them, but it appears to me some go to the opposite extreme. When I was Professor Becker's assistant in Heidelberg, fifteen years ago, our cataract cases were indeed subjected to a most trying ordeal. During the first foui or five days they were expected to lie prone on the back. Notwithstanding the impossibility of the occurrence of prolapsus, owing to the iridectomy done in every case, on the third or fourth day they were allowed to assume a half upright position, being supported by a back rest. At the end of a week one eye was unbandaged and the patient allowed to sit in a chair in a dark room. It required another week to accustom the operated eye to the light, which entered through a partially drawn Venetian blind. During the third week the patient was given the freedom of the hospital, the eye protected by smoked glasses. The middle course, not too much freedom and not too much restraint, I regard more in keeping with the demands of circumstances we are called upon to meet. The upper half of the cornea has been severed. This must re-unite. Any untoward movement of the eye or of the body which will tend to disturb the close adaptation of the flap, either on account of mechanical insult or increase of intra-ocular pressure, must be carefully avoided. This can best be accomplished by guarding the patient from such mishaps.

I do not intend to take up more of your time by entering into a detailed discussion of the various methods of after-treatment, but will, in conclusion, mention the method I invariably enforce. I always operate in the operating room, the patient reclining either in a chair or on an operating table. After extracting the cataract, both eyes are covered by pieces of clean linen dipped in boric acid. Over this are placed two thicknesses of thin cotton discs. The whole is kept in position either by a two inch broad gauze baudage, or more often by one made of starched mosquito bar netting. This latter material must not be starched too much, otherwise it will after drying, exert too much pressure on the eyes. The fine netting in commercial use will answer the purpose. After being. sterilized it is dipped in boric acid and applied. The patient is led to his room, and such clothes as have not been previously removed are now taken off. The parting instructions are, "Remain quietly on your back, or on the non-operated side for twelve hours." An aperient having been given the night before, the desire to evacuate the bowels will not arise until the third day. The bandage and wet cloths dry after a few hours and conform to the shape of the head and other parts they cover, thus exercising a uniform and gentle counter-pressure and rendering the eyes immobile. When the bandage is cut away it retains the form of the parts like a plaster-of-Paris dressing. Only fluid diet is allowed the first forty-eight hours. After twelve hours the patient is supported in a partially upright position by a back-rest. The eye is dressed for the first time on the third day unless subjective symptoms require an earlier inspection. After removal of the bandage (cut with scissors), the edges of the lids are washed with boric acid aud the eye inspected. Boric acid and atropin are dropped between the lids and the eye re-bandaged. This method of dressing the eye is repeated daily until the fifth day, when the eye is left open, the non-operated eye having been freed already on the third day after the operation. On the third day the patient leaves the bed and sits in a comfortable chair. On the seventh he leaves his room. This is my routine practice in the general run of cases. Strict adherence to the above method, which contemplates rapid union of the corneal wound, by moderate quiet of the patient and immobility of the eyes, appears to me to be responsible for the few cases (four) of prolapsus which have occurred in my practice. The presence of a well trained nurse helps greatly in reaching the desired end.

Permit me, in conclusion, briefly to review the main features of this paper. My object in reporting the eleven cases operated under atropin and with a periphery incision and in referring to other methods in vogue, is to demonstrate that good results are obtained, no matter where the corneal incision is placed; neither does the employment or disuse of eserin, atropin and cocain affect the occurrence of prolapsed iris. The most important factor in an ideal outcome is the after-treatment, the observance of methods which will counteract intra-ocular ten. sion and thus rapidly lead to reunion and firmness of the cornea.

\section{CLINICAL MICROSCOPY.} Read by title before the Tri-State Medical Society at St. Louis, April 2

\section{BY W. B. LA FORCE, PH.B., M.D.}

LECTERER ON PATHOLOGY AND OIRECTOR OF THE MICROSCOPIC AND IBACTERIOLOGIC LABORATORY OF THE KEOKUK (YOWA) MEDICAL COLLEGE.

In presenting a paper on the subject of scientific medicine and the microscope, I have no discoveries to reveal, nor shall I attempt to review the progress in the science of medicine brought about through the revelations of the microscope; but rather will I direct your attention to its clinical value. With this in view, we will omit detailed reference to the important facts revealed by studying the minute anatomy of the different organs in both their normal and diseased conditions. It is axiomatic that the more we know of the structure, both macroscopic and microscopic, of organs healthy or diseased, the more capa. ble are we to understand the physiologic and patho. logic processes which occur in those organs or tissues; and so we will not dwell on those points. Moreover a large part of such knowledge has been acquired through study of the post-mortem specimen.

But to-day I desire to emphasize the growing im. 
portance of the microscope in the clinic, or at the bedside, as an aid and sometimes as the all-essential factor in establishing an early and positive diagnosis and prognosis.

With the recent discoveries in chemistry, microscopy, and bacteriology, scientific medicine has probably advanced as much in the last few decades as in all previous time. The art of medicine is being replaced by the science of medicine. Not more than a generation ago, the successful practitioner must needs have had very extended observation of disease in order to weigh one symptom with another, and judge between them properly. Distinctive pathognomonic symptoms were not known so well as now. Diagnoses were more a matter of judgment, and naturally, other things being equal, the one who guessed the longest was the best guesser.

But at present, with a great many diseases, conditions have changed. Thorough laboratory courses may answer for several years of practice. Instead of looking for a combination of symptoms, we look for one distinctive symptom; and whereas the old practitioner, in many cases, after much observing and deliberation, conld give only a probable diagnosis, to-day, the young physician well drilled in chemistry, microscopy and bacteriology is able in many of the same cases to give an immediate and positive diagnosis and a more correct prognosis. The artist is giving way to the scientist, conjecture and judgment to confidence and knowledge.

The diseases which of ten can be positively diagnosed by a microscopic examination of the blood, and sometimes only by such an examination are: leukemia, pseudo-leukemia, oligo-cythemia, pernicious anemia, simple leucocy tosis, chlorosis, anthrax, miliary tuberculosis, glanders, typhoid fever, tetanus, relapsing fever and malaria, with its different tertian, quartan and atypical types; and those diseases caused by the animal parasites: distoma hematobium, and filaria sanguinis hominis.

To review a little, we find that oligo-cythemia is characterized by a decreased number of the red blood corpuscles without change in the number of the white. In health $1 \mathrm{cu} . \mathrm{mm}$. of blood contains according to Vierordt, four to five million red blood corpuscles. In oligo-cythemia, the number has been known to be decreased to 360,000 for the same volume. Probably the best means of determining the number for clinical use in diagnosis and in estimating the effect of therapeutic measures is the microscope, together with the Thoma-Zeiss counting apparatus. The amount of contained hemoglobin can be most accurately determined with Fleischl's hemometer.

The condition oligo-cythemia is present in simple anemia, chlorosis and pernicious anemia. In the simple anemia there is merely a decrease in the number of the red corpuscles with a normal amount of hemoglobin for each corpuscle. In chlorosis there is, beside the decrease in the number of the red corpuscles, also a decreased amount of hemoglobin in each corpuscle. While in pernicious anemia, a disease which is always fatal, there is, beside the les. sened number of red blood corpuscles and of their contained hemoglobin, the additional factor of poikilocytosis, i.e., a marked irregularity in their size and shape, with relative increase of the amount of hemoglobin.

Leucocytosis, on the other hand, is a condition characterized by an increase in the number of the blood's white corpuscles, which in health range from four thousand to ten thousand per cu. $\mathrm{mm}$.

A much more serious condition, which is generally fatal, and the diagnosis of which is therefore valuable, mainly for the prognosis, is leukemia, or leucocythemia, characterized by a great increase of the number of the white corpuscles, a decrease of the number of the red and of their relative hemoglobin contents, associated with a marked increase of ly mphoid tissue in the body. But pseudo-leukemia or Hodgkin's disease shows the increase of lymphoid tissue but not of the leucocytes.

Of the infectious diseases whose germs are sometimes found in the blood, those of anthrax, relapsing fever, and malaria may be recognized by microscopic examination of fresh blood; but in glanders, typhoid, tetanus and general miliary tuberculosis, cover-glass preparations of the blood must be made and stained before examined.

Given a suspected case of anthrax, anthrax edema, anthrax carbuncle, malignant pustule or woolsorter's disease, as the affection is variously called, an early diagnosis from acute phlegmonous inflammation and malignant edema is important, especially when the seat of origin is in the skin, for an early, and therefore thorough excision of the part may prevent general infection which latter when it occurs is generally fatal.

The diseases likely to be confounded with relapsing fever or its synonyms, bilious typhoid, famine fever, mild yellow fever, seven days fever, bilious relapsing fever, are atypical cases of malaria, sepsis, miliary tuberculosis, etc. But probably in nome of the infectious diseases is the exciting cause more readily demonstrated in the blood than is the spirillum Obermeier in the blood of those suffering from relapsing fever. In acute general miliary tuberculosis, always a fatal disease, the diagnosis not infrequently is difficult in the extreme. Typhoid fever, septicemia, pyemia, chronic local tuberculosis of the kidneys, complicated by uremia, non-tubercular meningitis, capillary bronchitis, and general carcinomatous or sarcomatous metastases are all similar in many points. The finding of bacillus tuberculosis in the blood, cover-glass preparations of which are made and stained in the same way as from sputum settles the question, both as to diagnosis and the inevitable prognosis.

The blood of typhoid patients is but rarely found to contain Eberth's bacillus, especially in the early stages of the disease, being found much earlier in the fecal evacuations. However, its demonstration in the blood would speak positively for the diagnosis and indicate a very serious condition of the patient.

Glanders seldom attacks the human species, but when it does the demonstration of the Rotz bacillus or bacillus Mallei is best done by staining the coverglass preparation according to Löffler's method. When the disease is purely local it may be confounded with syphilis or tuberculosis, but diagnosed and treated properly may be cured; however, when the germs have entered the circulation, a fatal ending is only a question of time.

Tetanus, although not a frequent disease in this climate, yet occasionally appearing as a wound disease, the discovery of the bacillus in the blood bids fair to assume considerable practical importance by reason of the discovery by Tizzoni and Cattani in the blood serum of an immune animal, of the tetanus antitoxin, analogous to Behring's diph theria antitoxin. 
In malarial affections we distinguish three distinct types of the disease: tertian, quartan, and irregular, and each is known to be accompanied by different forms of the plasmodium malariæ.

In the tertian type, which is the most common, the plasmodium or hemamœba malariæ requires two days for the growth of a generation, each attack of chill and fever marking the advent of a new generation and the parent is seen divided into fifteen to twenty segments.

In the quartan type, the parasite requires three days for its complete development and its progeny is six to twelve.

The atypical cases where the paroxysm is quite irregular, or even approaches the type of a remittent or continuous fever, or takes the form designated as chronic malarial poisoning, or malarial cachexia, is characterized most frequently by the half moon or sickle-shaped plasmodium of Laveran.

The pure intermittent quotidian is caused by three generations of the quartan type, each maturing on separate days.

Ordinarily, the pure tertian type, with the attack every other day, offers no great difficulty in diagnosis; but in the atypical forms already mentioned, a positive diagnosis is often long delayed and not infrequently impossible without the aid of the microscope.

From sputum and secretions and excretions of the oral cavity we recognize: parasitic stomatitis, tuberculosis pulmonalis, diphtheria or membranous croup, actinomycosis and croupous pneumonia.

In the examination of sputum for pulmonary tuberculosis, and of tonsillar or faucial exudates for the Klebs-Löffler bacillus of diphtheria, the microscope scores two of its greatest triumphs, the practical value of which is so well known that I shall not here dwell on them.

By examination of fecal discharge we may diagnose: cholera, typhoid fever, intestinal tuberculosis, and various animal parasites causing more or less of acute enteritis and the varieties under the subkingdom vermes as tænia, trichinæ, etc.

In microscopic examination of urine, much positive knowledge is obtained with reference to disease of genito-urinary organs, especially in differential diagnosis between simple hematuria, acute nephritis, chronic nephritis, contracted kidney and amygloid kidney, passive congestion of kidney and nephritic infarcts.

The form of predominating crystals in urinary sediment may reveal the nature of any existing calculi. Not infrequently, different tumors of the urinary tract, as papilloma, epithelioma and sarcoma shed small portions of their substance which are voided in the urine and whose nature can then be revealed best by the microscope.

Tuberculosis of the urinary tract can often be positively diagnosed only by cover-glass preparations of urinary sediments; and the seat of the ulcer, whether in the bladder, ureter or kidney, can often be determined by the predominance in the sediment of certain characteristic forms of epithelium desquamated by the ulcerous process.

Differentiation between a simple non-infective and gonorrheal urethritis is possible in all cases by the proper microscopic and bacteriologic methods, and in sume cases where all other methods would fail. Prostatorrhea, spermatorrhea and male sterility are also questions for the microscope to determine.
Finally, the finding in the urine of actinomycosis, ecchinococci, distoma hematobium, filarial sanguinis hominis and ascarides would explain otherwise very puzzling cases.

Of the cutaneous parasites, we recognize tricophyton tonsurans of ringworm, achorion Schoenlinii, of forms of ascoptes hominis of scabies, acarus folliculorum, beside the varities of pediculi.

Then in different varieties of abscess, or purulent exudates, we can often appeal with advantage to the microscope to determine whether it is caused by the common pyogenic germs, or the bacillus tuberculosis or, less frequently, by anthrax, lepra or tetanus bacilli, or by echinococci or actinomycosis.

Another very important field for microscopy is in the recognition and differential diagnosis of benign and malignant tumors, especially in parts suitable for operation; and in many cases, as has so frequently been stated already in this paper, the microscope offer's the only means of making a positive diagnosis early enough to allow of effective treatment.

Then in the domain of medical jurisprudence, where facts are demanded and conjecture is not allowed, the microscope plays an important rôle in the recognition of blood, hair, spermatozoa, etc.

In many of the conditions and diseases referred to, diagnosis can often be made with great probability, and even positively, by noting a combination of symptoms and observing the course of the disease, or by excluding the possibility of other diseases in the case. But such methods are always unsatisfactory and often dangerous in their delay. For some one or more of the combination of symptoms may remain absent or be slow in manifesting itself, the patient may die before the course of the disease is run, and diagnosis by simple exclusion is likewise a slow and unsatisfactory method. It is saying what the dis. ease is not, rather than what it is.

But the progress of medicine, as a science, demands and is achieving early and positive diagnoses. The single and distinctive pathognomonic symptom is being sought and found. The essential and exciting cause (without which the disease would not exist, no matter how many predisposing factors were operating) is being recognized, and the way better paved for the train of therapeutic measures to follow.

The hospital, with its clinical and bedside teaching, has always been and always will be essential in proper medical training. Laboratories are becoming so, more and more every day. They are making the clinics more available and practical; and those colleges with limited clinical facilities can not establish themselves as thorough and practical institutions without equipping and maintaining good laboratories, and with such they can become eminently practical and scientific, for they can then, and not till then, use their clinical material to the best advantage.

\section{CONCLUSIONS.}

1. Medicine in many points is fast becoming a practical science.

2. Microscopy and other laboratory methods are doing a great deal toward establishing the science of medicine.

3. Thorough drill of the student in laboratory methods may answer for several years of general practice.

4. Positive diagnoses and more correct prognoses are possible in a goodly number of diseases only by 
the microscope, and general practitioners not having had thorough training in this instrument of precision should, at least for the benefit of their patients, avail themselves of it, or call on the services of an expert in that line.

5. Medical colleges with limited clinical facilities may make their teaching eminently scientific and practical, by establishing and conducting good laboratories and thus get the best good from their clinics.

\section{PERITONEAL SUPPORTS-(LIGAMENTUM PERITONEI). \\ BY BYRON ROBINSON. \\ PROFESSOR OF GYNECOLOGY POST-GRADUATE SCHOOL. CHICAGO.}

(Continued from page 326.)

In my opinion to Meckel belongs the chief credit of the coalescence theory (1817) and Müller in 1830 simply confirmed his views and enlightened the world as to the origin and development of the great omentum. Yet it is not necessary to believe that even such celebrated men as Meckel and Müller could and duodenum. Hence one of Müller's errors is pointed out and corrected. Again, Müller noted that at first the mesogastrium arises in the mid-dorsal line, but with the change of the position of the stomach it becomes shifted toward the left. This is also an error of Müller, for the insertion of the mesogaster is always in the mid-dorsal line. Any one can prove that the mesogaster is always in the middorsal line by opening up an adult cat, when it will be found that the mesogaster has never varied from the mid-dorsal line. In adult man, it appears that the mesogaster is not in the middle line, but that is owing to a displacement of the serous membrane toward the left or, as the coalescence theorists would have it, the left blade of the mesogaster has coalesced with the dorsal parietal peritoneum. Hence, it is plain to observe two errors of Müller. Again he asserts that the coalescence (verklebung) between the anterior blade of the great omentum and the upper blade of the transverse mesocolon can be easily divided in embryos of the third and fourth month. Whether this be an error or not, I will say that I never found this condition of re-dividing or re-sep-

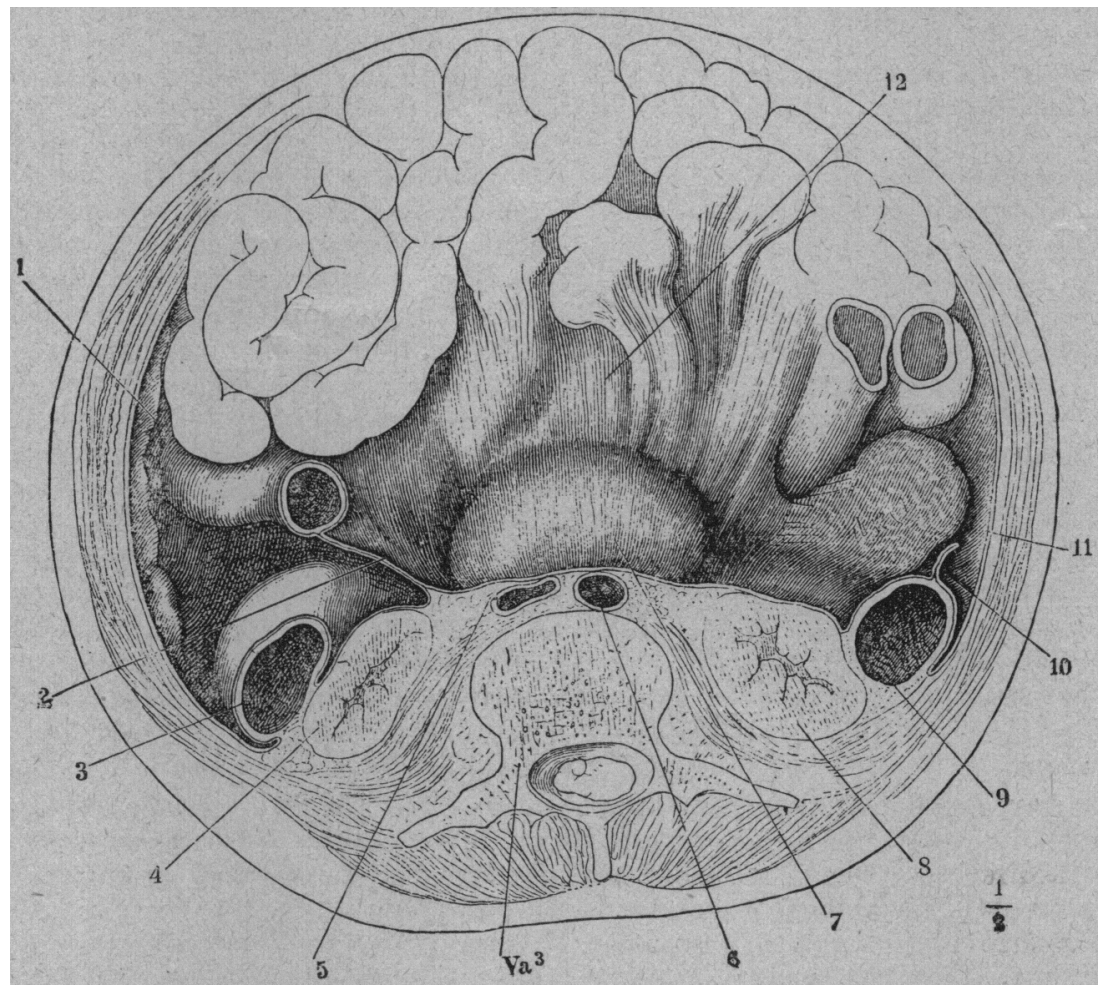

Fig. 39 (after Henle. 1873) represents a horizontal section of the abdomen between the third and fourth lumbar vertebre. The upper section, the small intestines, are spread out forward. 1 , border and under surface of the liver; 2 , section of the mesentery; 3 , colon dextrum; 4 , right kidney; 5 , vena cava; 6 , aorta; 7 , the lower transverse portion of the duodenum shim. mering through the mesentery; 8, left kidney; 9, colon sinustrum; 10, appendix epiploocus Henle, the greatest of modern anatomists, draws in the sketch no mesocolon, right or left.

not make mistakes. I have read with care and (arating the coalesced membranes possible in any pleasure Müller's article on the "Origin of the Great embryo of any age. Since we have found several Omentum," written in German in 1830. I find that palpable errors in Müller's noted essay of 1830, is it even Müller could labor under errors. For example, not possible that he was in error (and his followers) Müller states that the mesogaster ceases at the point when he announced the confirmation of Meckel's where the duodenum begins. Any one can easily theory of coalescence of the anterior blade of the disprove this statement by opening a human embryo great omentum with the upper blade of the transverse at six weeks or, still better, a dog or cat, whence in mesocolon? It must be admitted, however, that all three cases it will be plain and easy to see that when a very accurate and keen investigator like Toldt the mesogaster extends all the way down the of Vienna, lends his voice and pen to aid in supportduodenum to the junction with the ileum. The ing the theory of coalescence, it demands prudence mesogaster includes the mesentery of the stomach and caution in opposing it. Respectful attention to 